\title{
Philosophiques
}

\section{Antognazza, Maria Rosa. Leibniz : An Intellectual Biography, Cambridge, Cambridge University Press, 2009, 623 p.}

\section{Cameron Brown}

Volume 38, numéro 1, printemps 2011

URI : https://id.erudit.org/iderudit/1005734ar

DOI : https://doi.org/10.7202/1005734ar

Aller au sommaire du numéro

Éditeur(s)

Société de philosophie du Québec

ISSN

0316-2923 (imprimé)

1492-1391 (numérique)

Découvrir la revue

Citer ce compte rendu

Brown, C. (2011). Compte rendu de [Antognazza, Maria Rosa. Leibniz : An Intellectual Biography, Cambridge, Cambridge University Press, 2009, 623 p.] Philosophiques, 38(1), 340-343. https://doi.org/10.7202/1005734ar d'utilisation que vous pouvez consulter en ligne.

https://apropos.erudit.org/fr/usagers/politique-dutilisation/ 
blissement des règles du choc ", qui, passant en revue les contributions successives de Descartes, Newton, Leibniz et Huygens, s'interroge sur la manière dont la nécessité apodictique des mathématiques "peut se communiquer entièrement à la mécanique, par le biais d'une application sans reste de la géométrie au phénomène du mouvement, dans laquelle on n'aurait à postuler aucune propriété extrinsèque au concept mathématique des corps » (146-147). C'est ce questionnement, conduit historiquement, sur «la connexion des sciences au plan de leurs fondements rationnels et des concepts qu'elles manipulent effectivement» (132) qui définit et singularise, nous dit André Charrak, la position de l'empirisme franco-berlinois en philosophie de la connaissance.

L'espace nous manque pour rendre justice à maintes analyses développées dans l'ouvrage, au premier rang desquelles l'examen très serré de l'hypothèse foucaldienne du lien entre mathesis et genèse qui conduit l'auteur à mettre en garde contre les dangers d'un usage non critique du concept d'épistémè en histoire de la philosophie. Qu'on nous permette seulement de conclure en saluant le souci constant qui l'anime de situer l'empirisme dans le fil d'une histoire qui ne le réduise pas à trouver son sens et son achèvement dans une «apothéose» kantienne et qui permet à André Charrak d'en restituer la diversité, l'originalité et la fécondité.

VINCENT GUILLIN

Université du Québec à Montréal

\section{Antognazza, Maria Rosa. Leibniz: An Intellectual Biography, Cambridge, Cambridge University Press, 2009, 623 p.}

Le livre de Maria Rosa Antognazza est une contribution accomplie non seulement aux études leibniziennes, mais également à l'histoire intellectuelle moderne en général. Son succès principal est à trouver dans sa manière de relever le défi le plus intimidant des études leibniziennes: l'immense diversité, parfois démesurée, des intérêts intellectuels de Leibniz qui, jusqu'à récemment, avait «disloqué et divisé» (p. 3) le corpus leibnizien aux yeux des spécialistes, sans compter qu'elle obscurcissait notre image de l'homme derrière l'œuvre. En se basant sur le corpus toujours croissant d'études choisissant une cohérence à la fois temporelle et thématique dans l'œuvre leibnizienne, et notamment sur l'ouvrage de Christia Mercer (Leibniz's Metaphysics: Its Origins and Development, Cambridge, 2001), la combinaison adroite par Antognazza de récits historiques fluides et d'analyses textuelles pertinentes vient satisfaire le besoin d'une analyse globale - particulièrement nécessaire pour les lecteurs anglophones -, de la vie et de l'œuvre du savant allemand itinérant.

Dans son introduction, Antognazza souligne les quatre thèses méthodologiques qui guident son analyse. D'abord, loin d'être réduites à des pré- 
occupations pratiques ou théoriques, la vie et l'œuvre de Leibniz doivent être abordées comme un tout. Pour ce faire, Antognazza a esquissé avec soin les influences réciproques entre la pensée de Leibniz et ses circonstances concrètes. Ensuite, les divers intérêts, tant théoriques que pratiques, poursuivis par Leibniz sont tous traités heuristiquement en tant qu'ils sont unifiés par quelques principes de base, comme autant d'efforts contribuant à la gloire de Dieu et à l'amélioration de l'humanité. Les Demonstrationes Catholicae que projetait Leibniz, par exemple, «bien qu'elles n'aient jamais été réalisées dans leur entièreté et qu'elles changent de forme et de description selon différentes périodes, fournissent en quelque sorte un fil d'Ariane pour ceux qui souhaitent reconstruire l'unité sous-jacente à l'odyssée intellectuelle labyrinthique de Leibniz» (p. 90). Bien qu'elle semble parfois prendre grand soin d'affirmer cette unité programmatique, Antognazza s'efforce de s'en remettre à Leibniz lui-même qui a rarement manqué une occasion de promouvoir sa cause ou d'absorber un nouveau schème dans sa vision architectonique. En troisième lieu, on doit montrer que Leibniz s'était engagé envers ses principes de base à un stade très primitif de son développement intellectuel et qu'il n'y renoncera pas par la suite.

Le premier chapitre de l'ouvrage offre un portrait fascinant d'un jeune précoce se familiarisant rapidement avec la topographie intellectuelle allant de la période classique à la période moderne en lisant insatiablement les ouvrages de la bibliothèque érudite de son père. C'est dès ces premières lectures que Leibniz conçut pour la première fois, entre autres, la possibilité de construire une encyclopédie des sciences en général. Quatrièmement, les intentions programmatiques de Leibniz - tout particulièrement eu égard à l'œcuménisme ecclésiastique et au fédéralisme paneuropéen - font de lui un produit net de son environnement: l'effritement intellectuel, politique et théologique du Saint Empire romain germanique de la fin du XvII siècle. Ce point de vue est considéré comme un correctif vis-à-vis de la tendance à faire de Leibniz un grand philosophe ayant eu le malheur de languir dans l'arrière-pays européen. Le fait que ces thèses se supportent mutuellement est un signe de leur aptitude à satisfaire la tâche leibnizienne de restaurer l'unité dans la multiplicité.

Certains critiques de l'ouvrage discuté ici ont reproché aux thèses méthodologiques d'Antognazza de faire des "affirmations vagues et réservées ", particulièrement ceux qui postulent des axiomes ou engagements de base à l'intérieur du projet intellectuel global de Leibniz ${ }^{1}$. La critique consiste en ceci qu'en mettant trop d'emphase sur une continuité doctrinale robuste mais pourtant mal définie Antognazza sacrifie du coup une compréhension du caractère unique des textes individuels de Leibniz et de leurs préoccupations et contextes très spécifiques. Prenons par exemple le De Usu et Necessitate Demonstrationum Immortalitatis Animae de 1671, dans lequel

1. [En ligne: http://ndpr.nd.edu/review.cfm ? id=15446]. 


\section{2 • Philosophiques / Printemps 2011}

Leibniz se collette avec le mystère chrétien de la résurrection de la chair en proposant une substance corporelle activée par un principe quasi-spirituel interne, immatériel et indestructible, guidant les corps matériels dans le cours de leur développement, en déplaçant ultimement le fardeau de la résurrection actuelle vers le domaine de la métaphysique, de façon à ce que les corps ressuscitent seulement dans leur forme spirituelle. Selon la critique mentionnée, cette proposition pourrait être vue ou bien comme particulièrement similaire aux théories qui postulaient une certaine qualité occulte pour rendre compte de la croissance biologique, notamment celle des natures plastiques de Ralph Cudworth, ou bien comme contenant implicitement les germes de la conception monadologique tardive de la substance chez Leibniz. Toutefois, étant donné que la théorie monadologique tardive est considérablement différente de cette première conception de la substance corporelle, le choix d'Antognazza pour la deuxième interprétation est considéré comme vague et erroné. Si on met de côté le fait que Leibniz n'a pas lu Cudworth avant 1689 , le problème de cette critique est que les deux interprétations n'en sont pas moins mutuellement exclusives, et donc la méthode d'Antognazza n'empêche pas de souligner les emprunts de Leibniz à ses contemporains (et les influences qu'il a subies d'eux) afin de préserver la continuité doctrinale. En effet, le relevé dressé par Antognazza est enrichi d'épisodes dans lesquels des critiques précises ont forcé Leibniz à se colleter avec les points fondamentaux de ses différentes théories, comme lorsque le théologien jésuite René-Joseph Tournemine s'opposa à la doctrine de l'harmonie préétablie de Leibniz sur la base du fait qu'elle n'expliquait pas mieux l'union métaphysique entre le corps et l'âme que ne le faisait la théorie occasionnaliste à laquelle la conception de Leibniz était en partie censée s'opposer. Ce qui conduisit Leibniz à tenter d'appliquer la notion de vinculum substantiale à sa théorie des monades comme un type de lien métaphysique plus durable (p. 476-479). Ainsi, les continuités qu'Antognazza fait ressortir ne sont pas statiques: elle n'affirme pas que la théorie tardive était implicitement présente à l'esprit de Leibniz dans les années 1670, mais elle tente plutôt de montrer que ce sont des changements dynamiques sujets à des révisions fréquentes.

Un léger problème de l'ouvrage se trouve dans le fait qu'il y a souvent une disparité frustrante dans l'attention accordée à différents textes et correspondances, et ce, tout particulièrement dans les chapitres traitant de la période tardive de Leibniz. Par exemple, malgré le fait qu'elle prétend «ne pas trop s'attarder sur la présentation des textes bien connus» (p. 11), Antognazza consacre quand même douze pages (p. 406-418) au traitement des Nouveaux essais et à l'essai d'échange épistolaire de Leibniz avec Locke, alors que son échange avec le professeur flamand Burchard de Volder, indiscutablement aussi important, n'est traité que dans deux paragraphes seulement (p. 422-425). Et il est encore plus malheureux de voir Antognazza n'accorder qu'une seule phrase pour souligner le fait que Leibniz et Nicolaas 
Hartsoeker ont discuté de "problèmes de physique» et de l'existence des atomes (p. 474), même si elle inclut ce dernier à juste titre parmi les correspondants de la période de maturité de Leibniz qui méritent une "mention particulière». En faisant de telles omissions, Antognazza doit cependant être louée de n'avoir pas suivi les mêmes pratiques historiographiques que Leibniz, dont l'histoire des Guelfes ne s'est jamais émancipée de la pile de manuscrits sur son bureau.

En plus de notes de chapitre copieuses et d'une bibliographie détaillée de la littérature primaire et secondaire, l'ouvrage d'Antognazza comprend une table chronologique des événements importants dans la vie de Leibniz et un appendice contenant «la description par Leibniz de sa propre apparence physique, de ses habitudes et de son tempérament» (p. 557), avec la même description par Johann Georg Eckhart. Il comprend également un arbre généalogique de la famille d'Hanovre et deux cartes géographiques, une de l'Europe centrale et une autre des duchés de la Basse-Saxe du vivant de Leibniz.

Indépendamment de la position qu'on peut prendre dans les débats autour de l'érudition leibnizienne - ce que Antognazza a louablement évité —, Leibniz ajoutera décidément de la couleur et de la texture à notre appréciation du savant allemand. C'est une lecture recommandable aux étudiants qui s'intéressent non seulement à Leibniz mais aussi à l'ensemble de la philosophie du XVII ${ }^{\mathrm{e}}$ siècle.

CAMERON BROWN

Université Concordia

Schulthess, Daniel. Leibniz et l'invention des phénomènes, Paris, PUF, 2009, $291 \mathrm{p}$.

Le problème de la réalité des corps occupe une place centrale dans la pensée leibnizienne. D'une part, Leibniz s'accorde avec plusieurs philosophes de son époque pour affirmer que la science, en particulier la physique, doit exprimer les déterminations corporelles de manière mécanique: il s'agit d'expliquer la nature des corps depuis les propriétés de grandeur, de figure et de mouvement. Mais d'autre part, Leibniz défend une deuxième thèse qui le démarque de ses contemporains: l'essence des corps ne saurait se réduire à des déterminations mécaniques, puisque celles-ci relèvent de l'ordre des phénomènes. Les phénomènes physiques ne sont donc pas des propriétés ontologiques primitives, mais trouvent leur fondement dans des unités substantielles. Contre Descartes, Leibniz soutient que le corps, s'il est considéré comme res extensa, n'est pas une substance, mais plutôt une réalité phénoménale de degré ontologique second. L'une des difficultés de la position leibnizienne consiste dès lors à évaluer la nature métaphysique des phénomènes par rapport aux substances simples ou monades. 